\title{
Bifunctional Expression Vector Plasmid DNA-bi-shRNA EWS/FLI1 Type 1 Lipoplex
}

\author{
National Cancer Institute
}

\section{Source}

National Cancer Institute. Bifunctional Expression Vector Plasmid DNA-bi-shRNA

EWS/FL/1 Type 1 Lipoplex. NCI Thesaurus. Code C127121.

A proprietary plasmid DNA expression vector encoding bi-functional short hairpin RNAs (bi-shRNAs) targeting the identical type 1 translocation junction region of the human fusion oncogene Ewing sarcoma (EWS)/Ets family transcription factor Friend leukemia virus integration 1 (FLI1) and are encapsulated in liposomal delivery vehicle (lipoplex; LPX), with potential antineoplastic activity. pbi-shRNA EWS/FLI1 type 1 contains 2 stem-loop structures encoded by a plasmid vector: one cleavage-dependent unit with perfectly matched passenger- and guide-strand, which is the small interfering RNA (siRNA)-like component, and one cleavage-independent unit composed of a strategically mismatched double strand, which is the microRNA (miRNA)-like component. Upon intratumoral administration and transcription into tumor cells, one shRNA unit with an imperfectly matched sequence causes inhibition of EWS/FLI1 messenger RNA (mRNA) translation (through mRNA sequestration and cleavage-independent degradation) while the other unit with a perfectly matched sequence promotes EWS/FLI1 mRNA degradation (through cleavage-dependent mRNA silencing). This prevents EWS/FLI1 expression in tumor cells, which results in a reduction of tumor cell proliferation. The EWS/FLI1 type 1 fusion gene product is overexpressed in type 1 Ewing's sarcoma and correlates with increased tumor proliferation and poor prognosis. 